\title{
Supporting Information: Statistical properties of a slit-confined wormlike chain of finite length
}

\author{
Yue Teng, Nigel T. Andersen, and Jeff Z. Y. Chen* \\ Department of Physics and Astronomy, University of Waterloo, Ontario, Canada, N2L $3 G 1$
}

(Dated: August 16, 2021)

\section{SI1: PERTURBATION THEORY}

Here, we summarize the main steps of a first-order perturbation theory in $\epsilon$, used to carry out the calculation of $\left\langle\mathbf{R}_{x y}^{2}\right\rangle$ and $\left\langle\mathbf{R}_{z}^{2}\right\rangle$.

\section{A. $x y$-plane}

The addition of the auxiliary field $H^{\prime}$ to the original Hamiltonian requires solving the MDEs

$$
\begin{aligned}
\frac{2 \lambda}{L} \frac{\partial}{\partial t} q(z, \theta, \varphi ; t)= & {\left[\nabla_{\mathbf{u}}^{2}-2 \lambda \cos \theta \frac{\partial}{\partial z}\right.} \\
& +\epsilon \sin \theta \cos \varphi] q(z, \theta, \varphi ; t),
\end{aligned}
$$

and

$$
\begin{aligned}
-\frac{2 \lambda}{L} \frac{\partial}{\partial t} q^{*}(z, \theta, \varphi ; t)= & {\left[\nabla_{\mathbf{u}}^{2}+2 \lambda \cos \theta \frac{\partial}{\partial z}\right.} \\
& +\epsilon \sin \theta \cos \varphi] q^{*}(z, \theta, \varphi ; t)
\end{aligned}
$$

for the two propagators. Assume that these propagators are expanded in terms of $\epsilon$. To linear order,

$$
q(z, \theta, \varphi ; t)=q_{0}(z, \theta ; t)+\epsilon q_{1}(z, \theta ; t) \cos \varphi
$$

and

$$
q^{*}(z, \theta, \varphi ; t)=q_{0}^{*}(z, \theta ; t)+\epsilon q_{1}^{*}(z, \theta ; t) \cos \varphi .
$$

The subscript " 0 " refers to the solutions from the original MDEs where $\epsilon=0$. The $\cos \varphi$ factor is explicitly introduced such that the above MDEs reduce to a $\varphi$ indepedent form below. To order $\epsilon$, from (11) and (2), one needs to solve

$$
\begin{gathered}
\frac{2 \lambda}{L} \frac{\partial}{\partial t} q_{1}(z, \theta ; t)=\left[\frac{1}{\sin \theta} \frac{\partial}{\partial \theta} \sin \theta \frac{\partial}{\partial \theta}-\frac{1}{\sin ^{2} \theta}\right. \\
\left.-2 \lambda \cos \theta \frac{\partial}{\partial z}\right] q_{1}(z, \theta ; t)+\sin \theta q_{0}(z, \theta ; t)
\end{gathered}
$$

and

$$
\begin{array}{r}
-\frac{2 \lambda}{L} \frac{\partial}{\partial t} q_{1}^{*}(z, \theta ; t)=\left[\frac{1}{\sin \theta} \frac{\partial}{\partial \theta} \sin \theta \frac{\partial}{\partial \theta}-\frac{1}{\sin ^{2} \theta}\right. \\
\left.+2 \lambda \cos \theta \frac{\partial}{\partial z}\right] q_{1}^{*}(z, \theta ; t)+\sin \theta q_{0}^{*}(z, \theta ; t) .
\end{array}
$$

The initial and final conditions needed for solving these MDEs are $q_{1}(z, \theta ; t=0)=q_{1}^{*}(z, \theta ; t=1)=0$, respectively.

The two first-order functions are related to each other by the symmetry,

$$
q_{1}^{*}(z, \theta ; t)=q_{1}(z, \pi-\theta ; 1-t),
$$

which can be shown by direct substitution of the above into (6). Hence only one of the two MDEs needs to be solved. The density distribution function is also expanded in $\epsilon$,

$$
\rho(z ; \theta, \varphi)=\rho_{0}(z ; \theta)+\epsilon \rho_{1}(z ; \theta) \cos \varphi .
$$

To zeroth and linear orders,

$$
\rho_{0}=B \int_{0}^{1} \mathrm{~d} t q_{0}(z, \theta ; t) q_{0}^{*}(z, \theta ; t)
$$

and

$$
\rho_{1}=B \int_{0}^{1} \mathrm{~d} t\left[q_{0}(z, \theta ; t) q_{1}^{*}(z, \theta ; t)+q_{0}^{*}(z, \theta ; t) q_{1}(z, \theta ; t)\right] .
$$

The normalization constant $B$ is calculated from

$$
B^{-1}=2 \pi \int q_{0}(z, \theta ; t) q_{0}^{*}(z, \theta ; t) \sin \theta \mathrm{d} \theta \mathrm{d} z \mathrm{~d} t,
$$

because of the integral on $\varphi$, the contribution from the second term in (8) to $B$, vanishes. We then have,

$$
\begin{aligned}
\langle\sin \theta \cos \varphi\rangle & =\epsilon \int_{0}^{\pi} \mathrm{d} \theta \sin ^{2} \theta \int \mathrm{d} z \rho_{1}(z ; \theta) \int_{0}^{2 \pi} \mathrm{d} \varphi \cos ^{2} \varphi \\
& =\epsilon \pi \int_{0}^{\pi} \mathrm{d} \theta \sin ^{2} \theta \int \mathrm{d} z \rho_{1}(z ; \theta) .
\end{aligned}
$$

Finally,

$$
\left\langle\mathbf{R}_{x y}^{2}\right\rangle / 2 \lambda L=2 \pi \int_{0}^{\pi} \mathrm{d} \theta \sin ^{2} \theta \int \mathrm{d} z \rho_{1}(z ; \theta) .
$$

\section{B. z-direction}

A perturbation expansion on the auxiliary field $H^{\prime \prime}$ can be similarly made. The two MDEs now become

$$
\begin{array}{r}
\frac{2 \lambda}{L} \frac{\partial}{\partial t} q(z, \theta ; t)=\left[\frac{1}{\sin \theta} \frac{\partial}{\partial \theta} \sin \theta \frac{\partial}{\partial \theta}\right. \\
\left.-2 \lambda \cos \theta \frac{\partial}{\partial z}+\epsilon \cos \theta\right] q(z, \theta ; t),
\end{array}
$$


and

$$
\begin{array}{r}
-\frac{2 \lambda}{L} \frac{\partial}{\partial t} q^{*}(z, \theta ; t)=\left[\frac{1}{\sin \theta} \frac{\partial}{\partial \theta} \sin \theta \frac{\partial}{\partial \theta}\right. \\
\left.+2 \lambda \cos \theta \frac{\partial}{\partial z}+\epsilon \cos \theta\right] q^{*}(z, \theta ; t) .
\end{array}
$$

The two propagators are expanded, up to the linear order in $\epsilon$,

$$
q(z, \theta ; t)=q_{0}(z, \theta ; t)+\epsilon q_{1}(z, \theta ; t)
$$

and

$$
q^{*}(z, \theta ; t)=q_{0}^{*}(z, \theta ; t)+\epsilon q_{1}^{*}(z, \theta ; t) .
$$

To order $\epsilon^{0}$, the original MDEs are recovered. To order $\epsilon$, the propagators satisfy

$$
\begin{aligned}
& \frac{2 \lambda}{L} \frac{\partial}{\partial t} q_{1}(z, \theta ; t)=\left[\frac{1}{\sin \theta} \frac{\partial}{\partial \theta} \sin \theta \frac{\partial}{\partial \theta}\right. \\
& \left.\quad-2 \lambda \cos \theta \frac{\partial}{\partial z}\right] q_{1}(z, \theta ; t)+\cos \theta q_{0}(z, \theta ; t),
\end{aligned}
$$

and

$$
\begin{aligned}
-\frac{2 \lambda}{L} & \frac{\partial}{\partial t} q_{1}^{*}(z, \theta ; t)=\left[\frac{1}{\sin \theta} \frac{\partial}{\partial \theta} \sin \theta \frac{\partial}{\partial \theta}\right. \\
& \left.+2 \lambda \cos \theta \frac{\partial}{\partial z}\right] q_{1}^{*}(z, \theta ; t)+\cos \theta q_{0}^{*}(z, \theta ; t),
\end{aligned}
$$

whose initial and final conditions are $q_{1}(z, \theta ; t=0)=$ $q_{1}^{*}(z, \theta ; t=1)=0$.

The antisymmetry property

$$
q_{1}^{*}(z, \theta ; t)=-q_{1}(z, \pi-\theta ; 1-t) .
$$

can then be established by direct substitution of the expression to (19). This is in contrast to the symmetry property in (7). The use of the antisymmetry property allows us to calculate only one of the two propagators for the current problem.

The perturbation expansion of the density function gives,

$$
\rho(z ; \theta)=\rho_{0}(z ; \theta)+\epsilon \rho_{1}(z ; \theta) .
$$

The zeroth order density $\rho_{0}$ remains the same as in (9). The first-order function becomes

$$
\rho_{1}=C \int_{0}^{1} \mathrm{~d} t\left[q_{0}(z, \theta ; t) q_{1}^{*}(z, \theta ; t)+q_{0}^{*}(z, \theta ; t) q_{1}(z, \theta ; t)\right] .
$$

After taking the antisymmetry property into consideration, the normalization constant $C$ is given by

$$
C=B
$$

where $B$ is expressed in (11). Finally, to the linear order in $\epsilon$,

$$
\langle\cos \theta\rangle=2 \pi \epsilon \int \mathrm{d} \theta \mathrm{d} z \cos \theta \sin \theta \rho_{1}(z, \theta),
$$

which gives

$$
\left\langle\mathbf{R}_{z}^{2}\right\rangle / 2 \lambda L=2 \pi \int \mathrm{d} \theta \mathrm{d} z \cos \theta \sin \theta \rho_{1}(z, \theta) .
$$

\section{SI2: NUMERICAL METHODS TO SOLVE MDES}

Typically, the propagators $q_{0}(z, \theta, t)$ and $q_{1}(z, \theta, t)$ are functions of three variables, which are represented by the functions in a three-dimensional grid system. The range for $\tilde{z} / \tilde{H},[-1 / 2,1 / 2]$, is equally divided into $M+1$ grid points; the range for $\theta,[0, \pi], N+1$ grid points with an even $N$; the range for $t,[0,1], L+1$ grid points. The numerical solutions are found for progressive values of $L$, $N$ and $M$, up to $L=200000, N=100$ and $M=201$. For a typical calculated quantity, the final presented result is then extrapolated by an analysis of the numerical results as a function of $1 / L, 1 / N$, and $1 / M$.

The general form of the MDEs in this paper is

$$
\begin{aligned}
\frac{1}{\tilde{L}} \frac{\partial}{\partial t} q(\tilde{z}, \theta ; t)= & {\left[\hat{H}_{0}+\hat{H}_{1}+V(\theta)\right] q(\tilde{z}, \theta ; t) } \\
& +g(\tilde{z}, \theta ; t) .
\end{aligned}
$$

An Euler scheme is used to treat the dependence in the variable $t$, such that the initial condition $q(\tilde{z}, \theta ; t=0)$ is used to carry a calculation forward to the final $t=1$. The derivatives in the operator

$$
\hat{H}_{0}=\frac{1}{\sin \theta} \frac{\partial}{\partial \theta} \sin \theta \frac{\partial}{\partial \theta}=\frac{\partial^{2}}{\partial \theta^{2}}+\frac{\cos \theta}{\sin \theta} \frac{\partial}{\partial \theta}
$$

is represented by the central difference, except for $\theta=0$ and $\pi$. At these points, the boundary condition $\partial q / \partial \theta=$ 0 is used and L'Hôpital's rule converts the above operator to $\hat{H}_{0}=2 \partial^{2} / \partial \theta^{2}$. The convection-type operator

$$
\hat{H}_{1}=-\cos \theta \frac{\mathrm{d}}{\mathrm{d} \tilde{z}}
$$

is treated by the upwind scheme. The first-order derivative in $\tilde{z}$ is approximated by a forward or backward difference, depending on the sign of $\cos \theta$. In our case, the difference scheme is completely consistent with the BCs in the text, where only half of the $\theta$ space is specified with a $\mathrm{BC}$. To solve (5), a function

$$
V(\theta)=-1 / \sin ^{2} \theta
$$

is used. At $\theta=0$ and $\theta=\pi$, the function gives rise to a second-order singularity which would otherwise make the differential equation unstable. The requirement, that the derivative of the function and the function itself vanish at these boundary points, effectively converts this term into an operator, $-(1 / 2) \partial^{2} / \partial \theta^{2}$, to be added to $\hat{H}_{0}$.

The calculation of the properties in the $\tilde{L} \gg 1$ limit is performed based on a different numerical method, separately. As shown in the text, using the ground-state dominance representation, the propagator $q(\tilde{z}, \theta ; t)$ in (24) is replaced by a $t$-independent eigenfunction $\Psi(\tilde{z}, \theta)$, and the left-hand side of the partial differential equation is replaced by a term $-\mu \Psi(\tilde{z}, \theta)$, where $\mu$ is an eigenvalue. The difference representation of the operators on the right-hand side remains the same as explained above. The zeroth-order problem, for $\Psi_{0}(z, \theta)$ where $V(\theta)=0$, 
is numerically solved by the Implicitly Restarted Arnoldi Method [1, 2], as implemented by the $\mathrm{C}++$ software packages Eigen [3] and Spectra [4]. To solve for the firstorder function in Eq. (79) of the main text, $\Psi_{1}(z, \theta)$, now with a given $\mu$ and $\Psi_{0}(z, \theta)$, the entire equation is treated as a matrix inversion problem, in its finitedifference form. The LU decomposition method for matrix inversion is used, as implemented by Eigen [3].

\section{SI3: NUMERICAL SOLUTION OF THE PROPAGATORS}

There are two parameters in the numerical system, $\tilde{L}$ and $\tilde{H}$. The propagator functions produced for different set $[\tilde{L}, \tilde{H}]$ have similar symmetry features in their $\tilde{z}, \theta ; t$ space. As example, Fig. 1 displays the propagator functions for the case $[\tilde{L}, \tilde{H}]=[1,1]$.

The three plots in Fig. 11(a) represents $q_{0}(\tilde{z}, \theta ; t)$ at $t=0,1 / 2$ and 1 . On each plot, two different illustration methods are used. The first is a grey, three-dimensional wireframe plot where the vertical height represents the value of the function. The second is the projection of the function on the two-dimensional plane, $\tilde{z} / \tilde{H}$ and $\theta$, where the color represents the value of the function, given by the color bar on the right. One can observe that in Fig. 1(a), the symmetry $q_{0}(-z, \pi-\theta ; t)=q_{0}(z, \theta ; t)$ is maintained for all $t$, due to the original symmetry in the MDE and its BCs. This is consistent with the physical meanings of $q_{0}$ in a slit confinement.

Used for the calculation of $\left\langle\mathbf{R}_{x y}^{2}\right\rangle$, Fig. 1(b) are three plots of the first-order function $q_{1}(z, \theta ; t)$ at three different $t$ values. The function is initially set at $q_{1}(z, \theta ; t=$ $0)=0$. The three plots follow the same symmetry, $q_{1}(-z, \pi-\theta ; t)=q_{1}(z, \theta ; t)$, which is the same as that of $q_{0}$. Another notable feature is that $q_{1}(z, \theta=0 ; t)=$ $q_{1}(z, \theta=\pi ; t)=(\partial / \partial \theta) q_{1}(z, \theta=0 ; t)=(\partial / \partial \theta) q_{1}(z, \theta=$ $\pi ; t)=0$, as required to erase the divergence of the term $-1 / \sin ^{2} \theta$ at the specified $\theta$ values.

Used for the calculation of $\left\langle\mathbf{R}_{z}^{2}\right\rangle$, the first-order function $q_{1}(z, \theta ; t)$ is plotted at three different $t$ values in Fig. 1(c). By substitution, one can establish the antisymmetry, $q_{1}(-z, \pi-\theta ; t)=-q_{1}(z, \theta ; t)$, directly from Eq. (18). This can be clearly seen from the numerical solution in the second and third plots.

\section{SI4: THE LIMIT OF RODLIKE CHAIN}

The physical properties of a rodlike molecule in slit confinement can be calculated independently from the WLC formalism, as described below. The properties are compared with the WLC results in the limit of

$$
\tilde{L} \ll 1
$$

in the text, for any $\tilde{H}$.
First, we consider the case of $H \leq L$. The main effect of wall-confinement in half space is the reduction of the rotational space. When a rod center is located at a position, such that the center of mass has a distance $z \leq L / 2$ away from the wall, the contribution of the rotational degrees of freedom to the partition function is

$$
Q_{\text {rot }, \text { half }}=\int_{0}^{2 \pi} \mathrm{d} \varphi \int_{\alpha}^{\pi-\alpha} \mathrm{d} \theta \sin \theta=4 \pi \frac{z}{L / 2},
$$

where $\alpha$ is the angle between the rod and $\hat{z}$ when one end of the rod touches the wall. Including the translational degree of freedom, to the half space of the confinement, the partition function

$$
Q_{\text {half }}=A \int_{0}^{H / 2} \mathrm{~d} z Q_{\mathrm{rot}, \text { half }}=\pi \frac{A H^{2}}{L},
$$

where $A$ is the area of the system in the $x y$-direction. The confinement free energy, after accounting for the free energy zero, $-\ln Q_{\text {free }}=-\ln (4 \pi A H)$, is then

$$
\beta \Delta F=-\left[\ln \left(2 Q_{\text {half }} / Q_{\text {free }}\right)\right]=-\ln [H / 2 L] .
$$

For the second case, $H>L$, the entire $z$-space can be divided into $[0, L / 2],[L / 2, H-L / 2]$, and $[H-L / 2, H]$. The partition function is separately written in these three ranges,

$$
\begin{aligned}
Q & =\pi \frac{A(L / 2)^{2}}{L}+A(H-L) 4 \pi+\pi \frac{A(L / 2)^{2}}{L} \\
& =4 \pi A H(1-L / 2 H) .
\end{aligned}
$$

Taking the same free-energy zero, then the confinement free energy is

$$
\beta \Delta F=-\ln (1-L / 2 H) .
$$

The black curve in Fig. 2 of the text is plotted according to these formulae, which is a function of $H / L$ only.

The compression force $\Delta f$ is found from

$$
\beta \Delta f=-\beta \frac{\mathrm{d} \Delta F}{\mathrm{~d} H} .
$$

From the expressions of $\beta \Delta F$, one obtains

$$
L \beta \Delta f= \begin{cases}L / H & (\text { when } H \leq L) \\ (L / H)(2 H / L-1)^{-1} & (\text { when } H>L)\end{cases}
$$

which is plotted as the black curve in Fig. 3(c).

The mean square projection of the end-to-end distance on the $x y$-plane is also calculable. For $H \leq L$,

$$
\begin{aligned}
\left\langle\mathbf{R}_{x y}^{2}\right\rangle & =\frac{2 \pi A L^{2}}{Q_{\text {half }}} \int_{0}^{H / 2} \mathrm{~d} z \int_{\alpha}^{\pi-\alpha} \mathrm{d} \theta \sin \theta \sin ^{2} \theta \\
& =L^{2}\left(1-\frac{H^{2}}{6 L^{2}}\right)
\end{aligned}
$$


(a) $q_{0}$
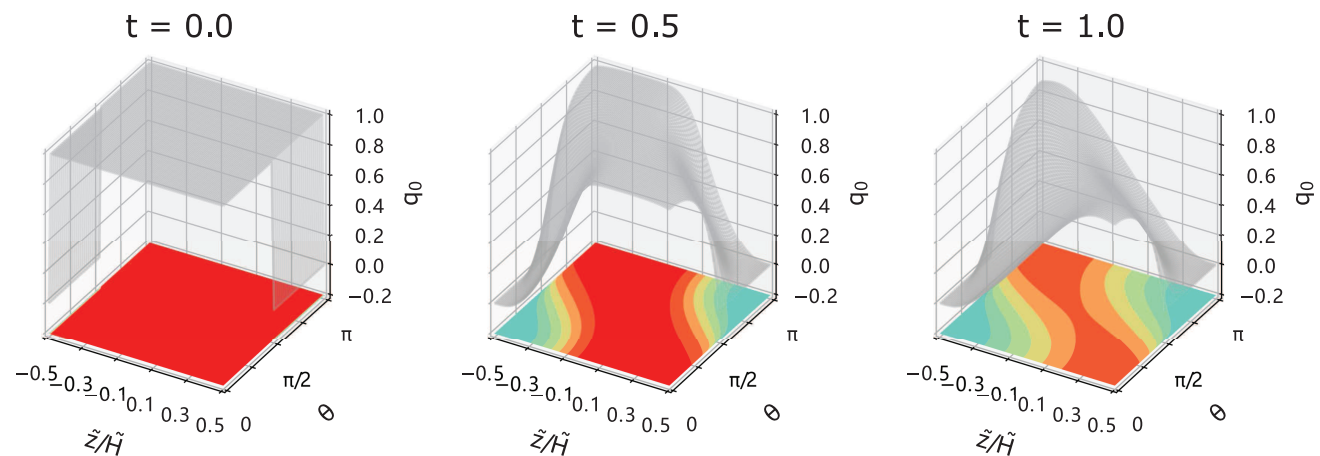

(b) $q_{1}$ in
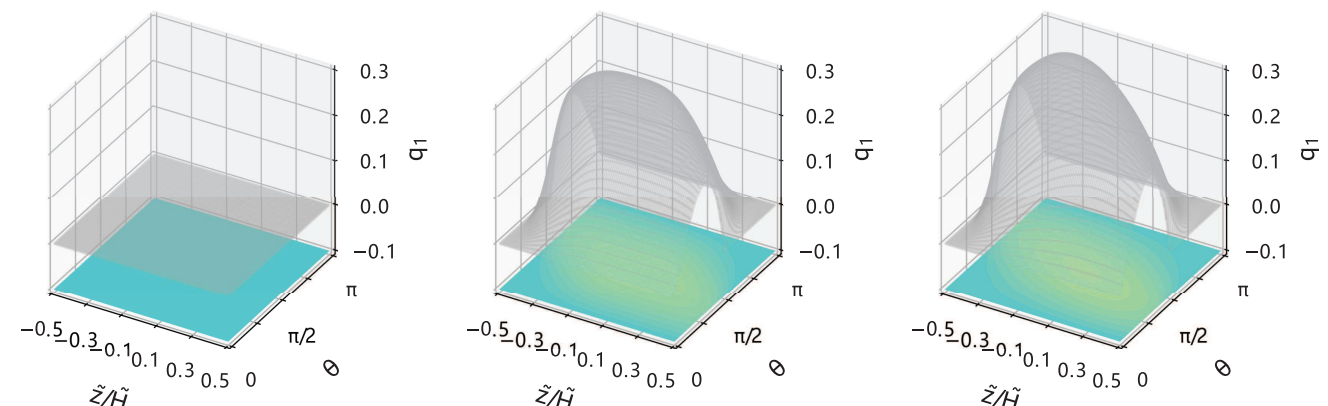

1.00

$\tilde{z} / \tilde{H}$

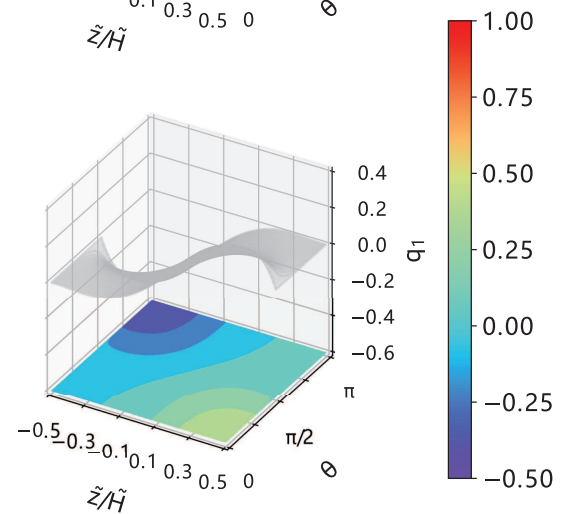

(c) $q_{1}$ in

eq. 18
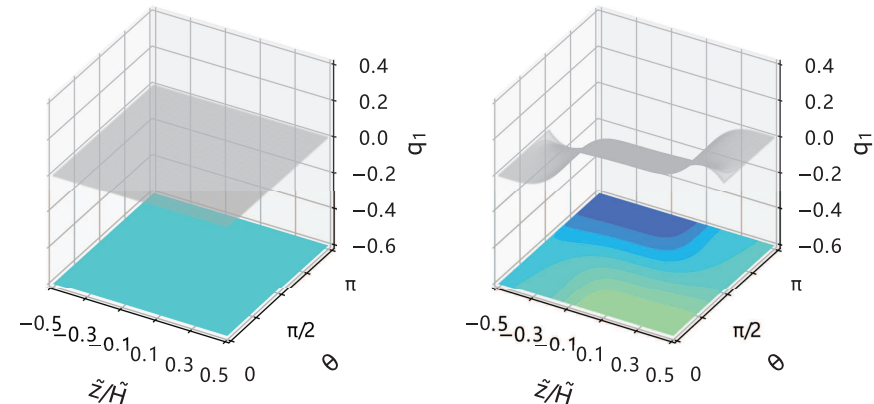

50

25

00

.25

0.50

FIG. 1. Symmetry properties of (a) the propagator function $q_{0}$, (b) its first-order perturbation function $q_{1}$ used for calculating $\left\langle\mathbf{R}_{x y}^{2}\right\rangle$, and (c) its first-order perturbation function $q_{1}$ used for calculating $\left\langle\mathbf{R}_{z}^{2}\right\rangle$. For a given set $[\tilde{L}, \tilde{H}]$, these functions generally depend on $\tilde{z}, \theta$, and $t$. The case $[\tilde{L}, \tilde{H}]=[1,1]$ is used here as example; other $[\tilde{L}, \tilde{H}]$ cases are qualitatively similar. The functions are plotted by two illustration schemes. The wireframe plot is a three-dimensional illustration of the function, given here in grey. The two-dimensional contour plot specifies the function value by the color bar on the right, on the $\tilde{z}-\theta$ base plane.

For $H>L$, again the entire $z$-region can be separated into three ranges as in the above,

$$
\begin{aligned}
\left\langle\mathbf{R}_{x y}^{2}\right\rangle=\frac{2 \pi A L^{2}}{Q} & {\left[\int_{0}^{L / 2} \mathrm{~d} z \int_{\alpha}^{\pi-\alpha} \mathrm{d} \theta+\int_{L / 2}^{H-L / 2} \mathrm{~d} z \int_{0}^{\pi} \mathrm{d} \theta\right.} \\
& \left.+\int_{H-L / 2}^{H} \mathrm{~d} z \int_{\alpha}^{\pi-\alpha} \mathrm{d} \theta\right] \sin ^{3} \theta \\
= & L^{2}\left(\frac{8 H / L-3}{12 H / L-6}\right) .
\end{aligned}
$$

The black curve, in Fig. 4 of the text, is plotted according to these analytic results.

For rodlike molecules, because $\left\langle\mathbf{R}^{2}\right\rangle=\left\langle\mathbf{R}_{z}^{2}\right\rangle+\left\langle\mathbf{R}_{x y}^{2}\right\rangle=$ $L^{2}$, we can take a short cut to calculate $\left\langle\mathbf{R}_{z}^{2}\right\rangle$. From the results above

$$
\left\langle\mathbf{R}_{z}^{2}\right\rangle= \begin{cases}H^{2} / 6 & (\text { when } H \leq L), \\ L^{2}(4 H / L-3)(12 H / L-6)^{-1} & (\text { when } H>L) .\end{cases}
$$

These are plotted as the black curve in Fig. 6 of the text. 
[1] Arnoldi, W. E. The principle of minimized iterations in the solution of the matrix eigenvalue problem. Quarterly of Applied Mathematics 1951, 9, 17-29.

[2] Lehoucq, R. B.; Sorensen, D. C. Deflation techniques for an implicitly restarted Arnoldi iteration. SIAM Journal on Matrix Analysis and Applications 1996, 17, 789-821.
[3] Guennebaud, G.; Jacob, B. , et al. Eigen v3. 2010; http://eigen.tuxfamily.org

[4] Qiu, Y.; Guennebaud, G.; Niesen, J. Spectra: C++ library for large scale eigenvalue problems. 2018; https://spectralib.org. 